\title{
Decision Support Systems: Present and Future
}

\author{
Lenka Lhotska ${ }^{1,2 *}$ \\ ${ }^{1}$ Czech Technical University in Prague, Czech Institute of Informatics, Robotics and Cybernetics, Prague, Czech Republic \\ ${ }^{2}$ Faculty of Biomedical Engineering, Czech Technical University in Prague, Prague, Czech Republic
}

\section{Abstract}

Background: Information and communication technologies have become inevitable and an almost inseparable part of our lives. They have also penetrated many application areas including medicine.

Objectives: The paper demonstrates the importance of correctly understanding the terms data, information and knowledge that represent the core of decision support and decision making processes.

Methods: In the paper we describe the development of decision support systems from the first generation using a simple knowledge representation up to complex distributed solutions.

Results: Based on the purpose, we identify three major groups of systems, namely recommendation, decision support and decision making systems.

Conclusion: We summarize the development of decision support systems and estimate the future directions.

\section{Keywords}

Decision support system; Recommendation system; Knowledge; Information; eHealth

\section{Correspondence to:}

\section{Lenka Lhotska}

Czech Technical University in Prague, Prague 6, Czech Republic E-mail: Ihotska@cvut.cz
EJBI 2018; 14(2):36-39

Received: March 20, 2018

Accepted: March 21, 2018

Published: April 30, 2018

\section{$1 \quad$ Introduction}

Healthcare is an information-intensive sector. Thanks to advanced technology, the number of devices connected directly to computers is continuously growing. Consequently, the volume of collected data is increasing tremendously. These new technologies are speeding up the exchange and use of data, information and knowledge, and are eliminating geographical and time barriers. These processes highly accelerated medical informatics development. Today's healthcare environments use electronic health records that are shared between computer systems and may be distributed over many locations and between organizations in order to provide information to internal users, to payers, and to respond to external requests. With increasing mobility of populations, patient data is accumulated in different places, but it needs to be accessible in an organized manner at national and even global scale. Large amounts of information may be accessed via remote workstations and complex networks supporting one or more organizations, and potentially this may happen within a national information infrastructure. The key terms in this context are data, information and knowledge [1], and decision support.

\section{Data, Information and Knowledge}

Data represents images of the real world in abstract sets. With the aid of symbols taken from such sets, data reflects different aspects of real objects or processes taking place in the real world. Mostly data are defined as facts or observations. Data results from a process of measurement or observation. Often it is obtained as output from devices converting physical variables into abstract symbols. Such data is further processed by humans or machines. Human data processing embraces a large range of options, from the simplest instinctive response to applications of most complex inductive or deductive scientific methods. Data-processing machines also represent a wide variety of options, from simple punching or magnetic-recording devices to the most sophisticated computers or robots.

The word information is often used without carefully distinguishing between different meanings it has taken on during its history. Generally, it refers to a finding or findings concerning facts, events, things, people, thoughts or notions, that is, a certain reflection of real or abstract objects or processes. It usually consists of its syntactic (structure), semantic (meaning), and pragmatic (goal) components. Therefore information can be defined as data that has been 
transformed into a meaningful and useful form for specific human beings. Data whose origin is completely unknown to us can hardly bring any information. We have to "understand" the data. An idea of where and under what conditions the data was generated is an important context of each message, and it has to be taken into account when we establish the information content of a message. Data sources thus become important components of what we are going to call information sources below.

The notion of knowledge is related to that of languageontology; within this model, the notion of knowledge can be treated in a more specific sense. This model is based on the assumption that there is the universe (ontology) consisting of objects which have certain properties and among which certain relations exist. Further we assume that there exists language in which ontology can be described. The notion of knowledge is mainly used intuitively. Within the model of language-ontology, its meaning can be defined in a more specific way. Knowledge will be understood as a true proposition concerning the ontology, formulated in the underlying language.

There are different kinds of knowledge. Analytical knowledge comprises those true propositions the validity of which can be derived from semantic postulates using purely logical means. It does not depend on the ontology, i.e., is valid in each and every one. Synthetic knowledge is knowledge, which is not true in every ontology and its validity must be decided on the basis of an experiment. Basic examples of synthetic knowledge include statements whose validity has been confirmed by direct observations. Such items of synthetic knowledge are called facts.

In practical applications we can find a large variety of systems that are described by their authors as expert systems, knowledgebased systems, or decision support systems. In fact however, some of them do not satisfy the basic characteristics of these systems. Some belong to classification or prediction systems, which are usually data intensive systems. Decision support systems can be characterized as knowledge intensive systems.

\section{Present and Future of Decision Support}

In the past, decision support systems were mostly used in narrow special applications and the users although not being top experts in the area had very good knowledge of it. The first generation of decision support systems was most frequently represented by simple, rule-based diagnostic system. From the programming technology point of view, they represented quite a specific class of decision support systems based typically on the inference net approach. Unfortunately, the simple first generation systems were not sophisticated enough to solve complex tasks in a wider problem area, most of all because they were limited to exploitation of the surface (shallow) knowledge, and the knowledge representation (based on the inference net, only) led to cumbersome behaviour of the system in the case the volume of relevant knowledge exceeded a certain threshold (if there were more than, let's say, 500 rules to be taken into account).
The advanced decision support system technology explored - besides the original idea of propagating uncertainty in an inference network - additional AI techniques which significantly enhanced the system capabilities. Specific methods and techniques have been developed to overcome the shortcomings of simple decision support systems mentioned above, e.g.:

- Different context/control links (having the nature of meta-rules) have been introduced to ensure both the common sense ordering of questions as well as the efficient cutting-off of irrelevant parts of knowledge bases in the early stages of a consultation run.

- Various blackboard control structures enable to split the knowledge into more knowledge bases (knowledge sources) and to cope with this set of knowledge bases in parallel. The WHEN-THEN rules are applied on the blackboard level to control the exploitation of the knowledge base set. Even a very simple blackboard control adds a lot of valuable features to the system. It enables, e.g., solving of monitoring tasks, problems requiring non-monotonic reasoning, zooming of attention, etc.

- Combination of different knowledge representation schemes has been performed with the stress to enable representation of causal, deep knowledge. It is possible to combine the inference net with the semantic/taxonomy net, with a subsystem for qualitative simulation, etc.

All the extensions and enhancements of "classical" rulebased diagnostic decision support systems described above represented steps towards more complex systems. The second-generation solutions considered separate knowledgebased modules of diverse nature, each being equipped by a specific inference algorithms. These modules were expected to be integrated by precisely specified, fixed links and relationships. The second generation decision support systems were able e.g. to integrate "classical" expert systems with neural net emulators, genetic algorithm based modules and databases. The information transfer among the modules enabling the system integration was ensured by a centralized integration program running in the integration framework.

Both the first and second generation decision support systems, even being significantly enhanced by very specific methods, operated very efficiently, but usually only in a narrow field of expertise. The next step in the development was connected with the rise of Internet and local as well as wide area networks allowing distributed solutions. Distributed problem solving and multi-agent architectures have become attractive for many application areas that are naturally distributed.

The multi-agent technology has already proven that it is suitable for creating an open, flexible environment able to integrate software pieces of diverse nature written in different languages and running on different types of computers. 
It enables to design, develop and implement a comparatively open multi-agent environment suitable for efficiently creating complex knowledge-based or decision support systems. Such an environment is able to integrate geographically distributed knowledge sources or problem solving units. Multi-agent systems have useful properties, such as parallelism, robustness, and scalability. Therefore they are applicable in many domains which cannot be handled by centralized systems. In particular, they are well suited for domains which require, for example, resolution of interest and goal conflicts, integration of multiple knowledge sources and other resources, time-bounded processing of very large data sets, or on-line interpretation of data arising in different geographical locations.

Modern health care is highly specialized. Complex examination of a single patient involves many expert consultations and laboratory tests. Medical knowledge, examinations and treatment are distributed functionally, geographically, and also temporally. There is a need for a reliable and consistent information flow among all participating subjects with the aim to satisfy the global goal - improved health of a patient. Of course, the necessary information flow is not predictable in extent and structure, but it develops and changes in time due to new knowledge and reactions. To satisfy these requirements and provide adequate decision support, the use of flexible intelligent software support is becoming increasingly desirable. Distributed problem solving and agent technology offer efficient and natural solutions, because they correspond to the main properties of the medical domain, namely distribution of information, problem-solving capabilities, resources, and responsibilities, decision-making with incomplete information, and iterative refinement of plans.

With the booming of information and communication technologies (ICT) and all smart devices, various applications appear having the characteristics of decision support. Health care is a typical example of an area in which the number of various applications is extremely high. However, we have to point out that only a fraction of these applications was verified by complex tests and satisfies requirements laid on software used in the medical environment. Therefore we have to distinguish these systems from several points of view: how they use knowledge; what their purpose is; who the primary users are.

Pharow P, et al. [2] discussed the main differences between three larger groups of systems that differ in the way how they use knowledge and information for decision support and decision making.

In summary, the three groups are:

1. Systems and devices that can be summarized as recommendation and suggestion systems, which are systems and devices we can find, for example, in the area of well-being and fitness, and which serve mainly for informing the users / clients about their health status;

2. Decision support systems - which serve as guidance for professionals and experienced users, like chronic patients on the one hand and active athletes on the other;
3. Decision making systems - which contains systems and devices that are directly linked with an actuator part influencing patient's health, by that way making decisions automatically or at least semi-automatically depending on, e.g., the severity of recorded and analyzed vital signs.

Additionally it is the human factor that is most significant, as the first two groups of applications require, and rely on, the interaction of a human being with a system, a machine, a device or an application. These two groups already represent, and will represent even more frequently in the future, the majority of applications directly used by patients.

The aforementioned division of systems is not the only one. We can differentiate the applications by the purpose into the following major groups. The first group aimed at clinical research uses the collected data in controlled manner. The application might be a pure software app or a combination of a medical device and software. The data are interpreted and verified by medical experts. The second group utilizing pure mobile applications developed in the frame of clinical research and transferred to routine use collects data from the patients. The data are sent for interpretation to a medical centre. The third group is aimed at the general population. The applications can be either mobile apps in smart phones or applications collecting data from, for example, a fitness wristband. Regarding the output of the applications, the first two groups might represent decision support systems, while the last group represents a recommendation system [2].

\section{Conclusion}

In the paper we tried to describe briefly the most important issues connected with decision support in medicine. Data, information and knowledge represent the core of decision making processes. During the last decades, decision support systems changed extremely from applications for narrow groups of specialists to more widely used applications. At the same time, it proved that the first generation systems were able to deliver expert decisions in a very narrow area of expertise, but failed outside the area and were not able to represent more complex knowledge. The second generation came up with new types of control structures and knowledge representation schemes. With the introduction of computer networks, distributed problem solving systems and successively multi-agent systems appeared and proved to be more flexible and efficient for tasks that are naturally distributed. Thanks to the availability of ICT, the systems are more widely spread and used. However, it is necessary to be aware of differences between various applications: they range from untested and unverified systems that can be only considered recommendation systems over decision support systems to decision making systems while both latter categories must be tested and verified for medical use.

The future development is clearly connected with advanced technologies, including utilization of the Internet of Things. 
Decision support and decision making based on applying advanced ICT in terms of micro and nano technology devices like sensors, actuators, and respective (wireless) body area networks (WBAN) will advance in the future.

\section{$5 \quad$ Acknowledgements}

Research has been supported by the AZV MZ CR project No. 15-25710A "Individual dynamics of glycaemia excursions identification in diabetic patients to improve self-managing procedures influencing insulin dosage".

\section{References}

[1]Zvárová J, Veselý A, Vajda I. Data, Information and Knowledge. In: Berka P, Rauch J, Zighed D. (eds.) Data Mining and Medical Knowledge Management. Information Science Reference. Hershey: IGI Global; 2009. p. 1-36.

[2] Pharow P, Lhotska L, Cheshire P. Personal Portable Devices as Enablers for Advanced pHealth Decision Support and Decision Making Services. Stud Health Technol Inform. 2013; 189: 27-32. 